\title{
GINS2 affects activity/differentiation, apoptosis and proliferation of osteoblast and osteoclast in steroid-induced osteonecrosis of the femoral head by regulating P53/ GADD45A signaling pathway
}

\author{
Lei TIAN ${ }^{1}$, Shui SUN ${ }^{1}$, Jian WANG ${ }^{1}$, Wei $\mathrm{LI}^{1}$, Xianquan WANG ${ }^{1 \star}$ (D)
}

\begin{abstract}
To investigate the function and mechanism of GINS complex subunit 2 (GINS2) in osteoblast and osteoclast in steroidinduced osteonecrosis of the femoral head (SINFH). Clinical specimens were collected from 40 cases of SINFH patients and 40 cases of control patients. Then, SINFH mouse model was built. Immediately, osteoblasts and osteoclasts were induced and differented from the pre-osteoblast cell line MC3T3-E1 and the mononuclear macrophage cell line RAW264.7 of mice, respectively. The qRT-PCR assay was used for detecting the expressions of GINS2, p53 and growth arrest and DNA damage 45A (GADD45A). The cytoactive, proliferation and apoptosis of osteoblasts and osteoclasts of SINFH mice were measured by tartaric acid phosphatase staining, CCK-8 and Annexin V-FITC/PI dual staining, respectively. GINS2 was up-regulated while p53/GADD45A was down-regulated in femoral tissues in SINFH patients and mice. GINS2 was down-regulated while p53 and GADD45A were up-regulated in osteoblasts of SINFH mice, which presented opposite trends in osteoclasts formations. GINS2 decreased osteoblasts activities and increased osteoclasts activities, inhibited apoptosis and promoted proliferation in osteoblasts and osteoclasts via p53/GADD45A pathway. GINS2 affects activity/differentiation, apoptosis and proliferation of osteoblast and osteoclast in steroid-induced osteonecrosis of the femoral head by regulating p53/GADD45A signaling pathway.
\end{abstract}

Keywords: GINS2; osteoblasts; osteoclast; p53/GADD45A pathway; SINFH.

Practical Application: GINS2 may be a potential target for diagnosis and treatment of SINFH.

\section{Introduction}

Osteonecrosis of the femoral head (ONFH) is a common refractory disease in the department of orthopedics leading to femoral head collapse and hip dyskinesia (Wang et al., 2019; Seixas et al., 2020). ONFH includes two types: non-traumatic ONFH and traumatic ONFH (Amanatullah et al., 2011; Mont et al., 2006). High incidence of non-traumatic ONFH is occurred in young and middle patients who aged 30-50 years, and the pathogeny of non-traumatic ONFH is multifactorial including hereditary susceptibility and risk factors for morbidity such as prolonged intensive use of hormones, alcohol, connective tissue disease, or hematologic illness (Amanatullah et al., 2011; Mont et al., 2006; Chen et al., 2019). The etiology of traumatic ONFH is clear, including fracture and dislocation, etc (Mont et al., 2006). Among them, steroid-induced ONFH (SINFH), as an iatrogenic disease, the incidence of which is increasing with the years and usually develops at an early phase in the procedure of steroid administration (Zhang et al., 2013; Yu et al., 2019). The pathogenesis of ONFH is still misty, and the highly effective treatment for it is not appeared by now (Rajpura et al., 2011; Wu et al., 2019). Moreover, the treatment methods of SINFH at clinical are mainly marrow core decompression bone grafting, intervention, traditional Chinese medicine, bone marrow stem cell transplantation, artificial hip replacement and so on, but the fundamental prevention and treatment strategy of SINFH is lacking. Thereby, further exploring the pathogenesis of SINFH to look for new therapeutic targets of it is very urgent.
It has been well known that osteoblasts and osteoclasts are the two main functional osteocytes in bone reconstruction, they can interact to each other. Adjusting the proportion of production and biological activity of osteoblasts and osteoclasts, which can regulate the dynamic balance of bone resorption and bone formation, thus affecting bone metabolism (Atanga et al., 2011; Park et al., 2010; Lee et al., 2003). Normally, there is a dynamic balance between osteoblast-mediated bone formation and osteoclast-mediated bone resorption, while this dynamic balance is broken, it will causes various degrees of bone remodeling abnormalities, resulting in bone metabolic diseases such as ONFH, osteoporosis, osteoarthritis, etc (Wang et al., 2015). Hence, maintaining the proportional balance between osteoblasts and osteoclasts may be one of the pivotal factors in the treatment of ONFH. Previous study has reported that the promotion of osteoblast activity and inhibition of osteoclast activity contribute to the improvement of ONFH (Tian et al., 2020). Furthermore, proosteoblast (bone progenitor cell) can differentiate into osteoblast and mononuclear macrophage can differentiate into osteoclast, and osteoblast is need to undergo four stages of osteoblast proliferation, extracellular matrix maturation, extracellular matrix mineralization and osteoblast apoptosis during bone formation. It has been proved that low-expression of GINS complex subunit 2 (GINS2) plays a inhibitory role in cell proliferation and stimulative role in cell apoptosis by regulating $\mathrm{p} 53$ /growth arrest and DNA damage 45A (GADD45A) pathway (Chi et al., 2020). However, whether 
GINS2 adjusting p53/GADD45A pathway to play roles in proliferation and apoptosis even activity/differentiation of osteoblast and osteoclast of ONFH is unreported. Therefore, in this work, we aimed to explore the roles of GINS2 in activity/ differentiation, apoptosis and proliferation of osteoblast and osteoclast in SINFH via regulating p53/GADD45A signaling pathway, then to provide possible molecular targets for diagnosis and treatment of SINFH.

\section{Materials and methods}

\subsection{Clinical specimens}

40 cases of patients diagnosed with SINFH (21 males, 19 females, age of 26-82 y with mean age 61.23 y) and 40 cases of control patients diagnosed with fresh femoral neck fractures (17 males, 23 females, age of 45-80 y with mean age $61.67 \mathrm{y}$ ) who suffered hip replacement surgery in our hospital from April 2015 to April 2019 were enrolled. The operations of all groups patients were implemented by the same group of doctors and the control patients were underwent surgery within 1 week after the injury. The basic data including age and gender presented no significant differences between two groups of subjects. The tissues at fracture surface of the head of femur were collected as specimens. An overtly necrotic area of specimens were selected to take as target necrotic bone mass specimens. The research design was approved by the ethics committee of our hospital and followed the tenets of the Declaration of Helsinki, and the informed consent of the sufferers or /and their family members was obtained and the informed consent to anesthesia was signed before the operation.

\subsection{SINFH mouse model establishment}

20 healthy C57BL/ 6 female mice with 6 month old and clean grade were purchased from Beijing Viton Lihua Experimental Animal Technology Co., Ltd. All mice were raised for at least 1 week in a temperature-controlled room at $25^{\circ} \mathrm{C}$ and $60 \%$ relative humidity with 12 hours' light cycle and given free access to standard laboratory diet and water before experiments. The mice were treated according to the ethical guidelines of the animal center, and the Animal Studies Committee approved the experimental protocol. Mice were randomly divided into 2 groups: normal control group $(\mathrm{NC}, \mathrm{n}=10)$ and the model group $(\mathrm{n}=10)$. The mice in the model group were received intramuscular injection of Prednisolone Acetate $(49 \mathrm{mg} / \mathrm{kg}$ ) while the mice in the NC group were received equivalent saline injection daily with once a day for $6 \mathrm{~d}$ (Tian et al., 2020). On the 7th day of model establishment, all mice were executed by breaking neck, and the femoral tissues were taken and reserved in ultralow freezer at $-80^{\circ} \mathrm{C}$ for later use.

\subsection{Cell culture and differentiation induction}

The mouse preosteoblast line MC3T3-E1 and mouse mononuclear macrophage line RAW264.7 were provided by the Cell Bank of Chinese Academy of Sciences (Shanghai, China). Preosteoblast and mononuclear macrophage were separated out from two groups of femoral tissues of mice and were cultured and passaged according to the methods of MC3T3-E1 and RAW264.7 cells under manufacturer's guide. All cells were maintained in Dulbecco's Modified Eagle's
Medium (DMEM; Sigma-Aldrich, St Louis, MO, USA) with 10\% fetal bovine serum (FBS) (Gemini Bioproducts, Calabasas, CA) and $1 \%$ penicillin and streptomycin (Sigma-Aldrich, Castle Hill, NSW, Australia) in a $5 \% \mathrm{CO} 2$ incubators at $37{ }^{\circ} \mathrm{C}$ for realizing cell culture. For osteoblasts differentiation induction, osteogenic differentiation inducers $(10 \mathrm{mmol} / \mathrm{L}$ of natrium glycerophosphoricum, $10 \mathrm{nmol} / \mathrm{L}$ of dexamethasone and $50 \mu \mathrm{g} / \mathrm{mL}$ of ascorbic acid) were added into MC3T3-E1 culture medium and the culture medium was replaced termly. The time of differentiation induction was 3 weeks. For osteoclasts differentiation induction, the inducers of RANKL100 ng/mL was added into RAW264.7 culture solution and the culture solution was replaced termly. The differentiation induction was performed for 1 week.

\subsection{Cell transfection}

After the differentiation - induced osteoblasts and osteoclasts from model group in the logarithmic growth period were uniformly seeded into 6-well plates with serum-free medium at $2 \times 10^{5}$ cells/well concentration overnight. Then, a short interfering RNA (siRNA) targeting GINS2(siRNA-GINS2), p53(siRNA-p53), GADD45A (siRNA-GADD45A), and siRNA negative control (NC) which purchased from Shanghai Gene Chem (Shanghai, China), as well as plasmid $(100 \mathrm{nM})$ were transfected into cells using Lipofectamine ${ }^{\mathrm{TM}} 2000$ transfection reagent (Invitrogen, USA) according to the manufacturer's guidances for at least $5 \mathrm{~h}$ at $37^{\circ} \mathrm{C}$ before the medium was replaced with fresh medium.

\subsection{Real-time quantitative reverse transcription-polymerase chain reaction ( $q R T-P C R)$}

qRT-PCR was carried out to quantify the mRNA expression levels of GINS2, p53 and GADD45A in clinical specimens, mice femoral tissues and diverse osteoblasts and osteoclasts referencing the previous report (Chen, 2018). Briefly, total RNA was extracted and isolated using Trizol reagent (Invitrogen, USA) according to the manufacturer's instructions. After cDNAs were synthesized by using high-capacity cDNA Reverse Transcription Kit (Applied Biosystems, USA). The relative mRNA expressions of GINS2, p53 and GADD45A were quantified by using the PrimeScript ${ }^{\circledR}$ RT reagent Kit (TaKaRa, Dalian, China). GAPDH was used as an internal control. The relative quantification was measured by the $2^{-\Delta \Delta C T}$ method. The PCR assays were repeated for three times.

\subsection{Activity testing}

For osteoblasts activity testing, the differentiation inducers treated-MC3T3-E1 cells were fixed with 4\% paraformaldehyde for 30 min and washed with phosphate buffer solution (PBS) for 3 times. Then, bone alkaline phosphatase (BALP) staining was performed according to the instructions of alkaline phosphatase (ALP) kit (Sigma, USA). Then, cells were washed and incubated for $15 \mathrm{~min}$ at room temperature in dark. After the chromogenic reaction was stopped, the cells were placed under optical microscope (Japan Olympus Corporation) for observation. For osteoclasts activity testing, RAW264.7 cells were collected and placed in a 96-well plate to adjust the cell density to $1.50 \times 10^{4} /$ well. After RAW264.7 cells were inducted and cultured for 1 week in the DMEM medium (containing RANKL $100 \mathrm{ng} / \mathrm{mL}$ ), the tartrate-resistant acid phosphatase (TRAP) staining was performed according to the instructions of TRAP kit (Sigma, USA) and RAW264.7 cells were rinsed 3 times 
with PBS. Then, the RAW264.7 cells were fixed for 30 min with $4 \%$ paraformaldehyde and washed with PBS for 3 times followed by permeated, rinsed and incubated. Then, staining solution was added and reacted for $60 \mathrm{~min}$ followed by observed under optical microscope. The activities of osteoblasts and osteoclasts were measured according to the staining light intensity.

\subsection{Cell proliferation assay}

A cell counting Kit-8 kit (CCK-8; Beyotime, Shanghai, China) was used to assay cell proliferation following the manufacturer's instructions. Briefly, differentiation - induced osteoblasts and osteoclasts from model group were seeded in 96-well plates at $5 \times 10^{3} /$ well and incubated for $3 \mathrm{~h}$ at $37^{\circ} \mathrm{C}$ with $10 \mu \mathrm{L}$ CCK- 8 solution in $100 \mu \mathrm{L}$ fresh media. The optical density was read at an absorbance wavelength of $450 \mathrm{~nm}$ at $0 \mathrm{~h}, 24 \mathrm{~h}, 48 \mathrm{~h}, 72 \mathrm{~h}$ and $96 \mathrm{~h}$ using a full wavelength microplate analyzer (Molecular Devices). Each result was the mean of triplicate assays.

\subsection{Annexin V-FITC/PI dual staining}

After transfection, cells from each group were performed Annexin V-fluorescein isothiocyanate (FITC, ab14085; Abcam)/ propidium iodide (PI, Beyotime, Beijing, China) dual staining for detecting cell apoptosis. The apoptosis rate was gauged with FACSCalibur flow cytometer (FCM, Becton Dickinson, Franklin Lakes, NJ, USA. The specific operational steps and description were referred to previous study (Chi et al., 2020).

\subsection{Statistical analysis}

Statistical software SPSS 16.0 (SPSS, Chicago, IL, USA) was adopted for statistical analysis, and the outcomes were expressed as means \pm standard deviation (SD). To assess statistical differences, quantitative data between two groups were assessed using the independent-samples t-test and Student's t-test. For all continuous data, normality tests were performed. Comparing the percentage/rate data between groups were evaluated with $\mathrm{X} 2$ test, non-parametric Mann-Whitney U test, and Fisher's exact test. Differences were considered statistically significant as $\mathrm{P}<0.05$.

\section{Results}

\subsection{The expressions of GINS2, p53 and GADD45A in clinical specimens}

qRT-PCR results showed that GINS2 was up-regulated while p53 and GADD45A were down-regulated in SINFH clinical specimens compared to the control tissues $(\mathrm{P}<0.01)$ (Figure 1 ).

\subsection{The expressions of GINS2, p53 and GADD45A in femoral tissues of mice}

qRT-PCR results displayed that GINS2 was up-regulated while p53 and GADD45A were down-regulated in femoral tissues of SINFH model mice compared to the control group $(\mathrm{P}<0.01)$ (Figure 2).
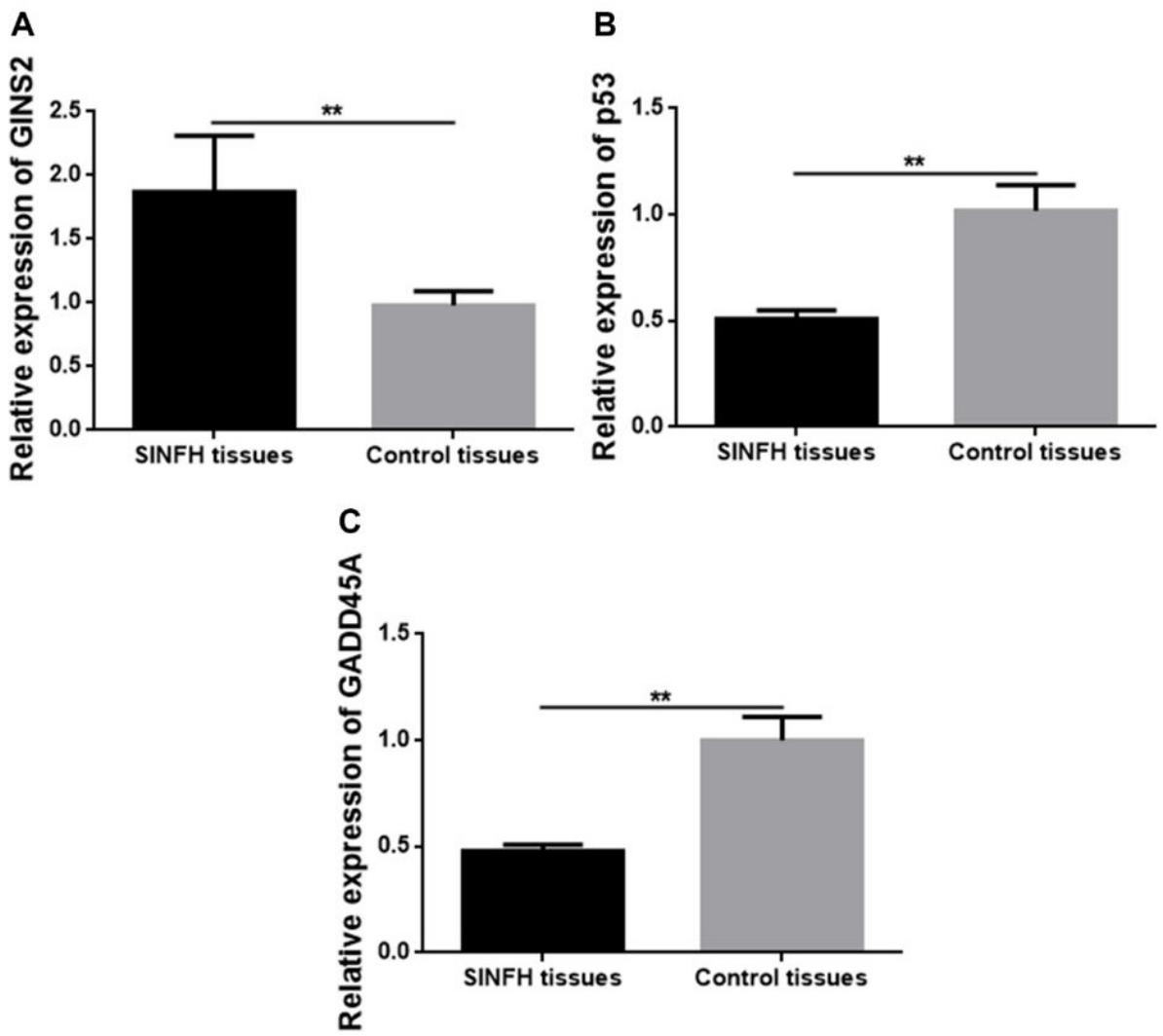

Figure 1. The relative mRNA expressions of GINS2, p53/GADD45A in clinical specimens. GINS2, GINS complex subunit 2; GADD45A, growth arrest and DNA damage 45A; SINFH, steroid-induced osteonecrosis of the femoral head; ${ }^{* *}$, vs Control tissues, $\mathrm{P}<0.01$. 
A

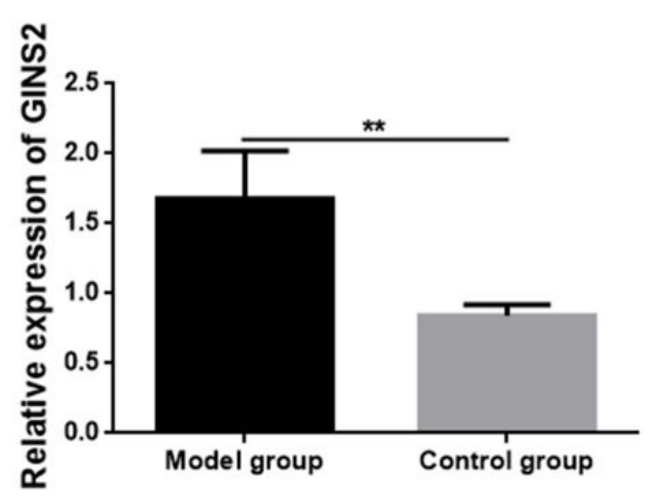

B

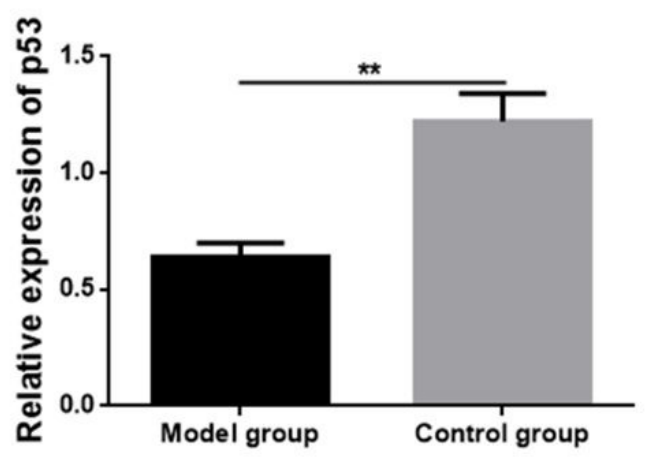

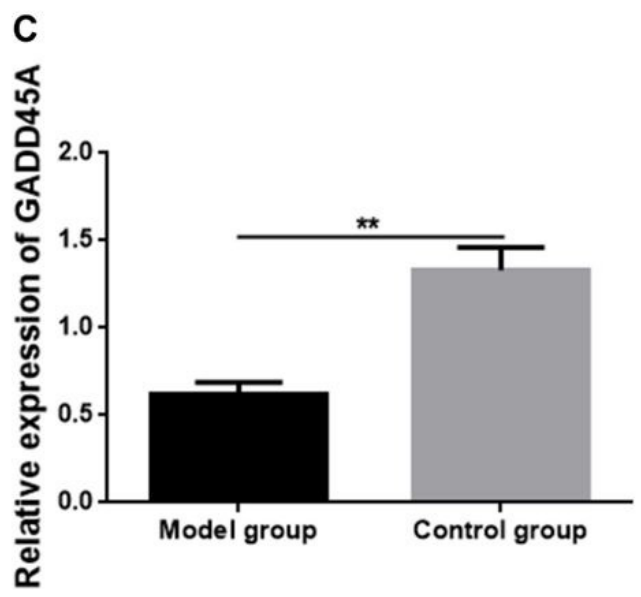

Figure 2. The relative mRNA expressions of GINS2, p53/GADD45A in femoral tissues of mice. GINS2, GINS complex subunit 2; GADD45A, growth arrest and DNA damage $45 \mathrm{~A} ;{ }^{* *}$, vs Controlgroup, $\mathrm{P}<0.01$.

\subsection{The expressions of GINS2, p53 and GADD45A in osteoblasts and osteoclasts of mice}

qRT-PCR results revealed that the expression of GINS2 was significantly down-regulated while p53 and GADD45A were evidently up-regulated in the process of osteoblasts formations that differentiated from mouse pre-osteoblast line MC3T3-E1 in the SINFH model group compared with that in the control group. Meanwhile, in the procedure of osteoclasts formations that differentiated from mononuclear macrophage line RAW264.7 in SINFH model mice, GINS2 presented a marked upward trend while p53 and GADD45A showed a visible downward tendency compared to the control group $(\mathrm{P}<0.01)$ (Figure 3$)$.

\section{4 siRNA-GINS2 led to a down-regulation of GINS2} expression and up-regulated expressions of $p 53$ and GADD45A in osteoblasts and osteoclasts of SINFH model mice

Compared to the siRNA-NC, siRNA-GINS2 resulted in a significant reduction in GINS2 expression and significant increases in $\mathrm{p} 53$ and GADD45A expressions in osteoblasts and osteoclasts of SINFH model mice detected out by qRT-PCR $(\mathrm{P}<0.01)$ (Figure 4).
3.5 Knockdown of GINS2 expression increased osteoblasts activities and decreased osteoclasts activities, promoted apoptosis and inhibited proliferation in both osteoblasts and osteoclasts via promotion of p53/GADD45A signalling

In this work, we detected the activities of osteoblasts and osteoclasts to reflect the situation of osteoblasts and osteoclasts formations which differentiated from MC3T3-E1 and RAW264.7 cells of the SINFH mice, respectively. The activities of osteoblasts were enhanced while the osteoclasts activities were weakened in siRNA-GINS2 group compared with siRNA-NC group $(\mathrm{P}<0.01)$ (Figure 5A), and after cotransfected into siRNA-p53 or siRNA-GADD45A, the enhanced or weakened trends were overturned partially $(\mathrm{P}<0.05)$ (Figure $5 \mathrm{~A})$. Compared to siRNANC group, siRNA-GINS2 caused a significant rise in apoptosis rate in osteoblasts and osteoclasts of SINFH model mice $(\mathrm{P}<0.01)$ (Figure 5B), however, cotransfection of siRNA-p53 or siRNAGADD45A, the increased tendencies were overturned partially $(\mathrm{P}<0.05)$ (Figure 5B). Osteoblasts and osteoclasts proliferation abilities in siRNA-GINS2 group were distinctly degressive compared with siRNA-NC group $(\mathrm{P}<0.01)$ (Figure $5 \mathrm{C}, 5 \mathrm{D})$, and osteoblasts and osteoclasts proliferation capabilities in siRNA-GINS2+siRNA-p53 or siRNA-GINS2+siRNA-GADD45A group were distinctly elevated on 4 th day compared with siRNAGINS2 group $(\mathrm{P}<0.05)$ (Figure 5C, 5D). 

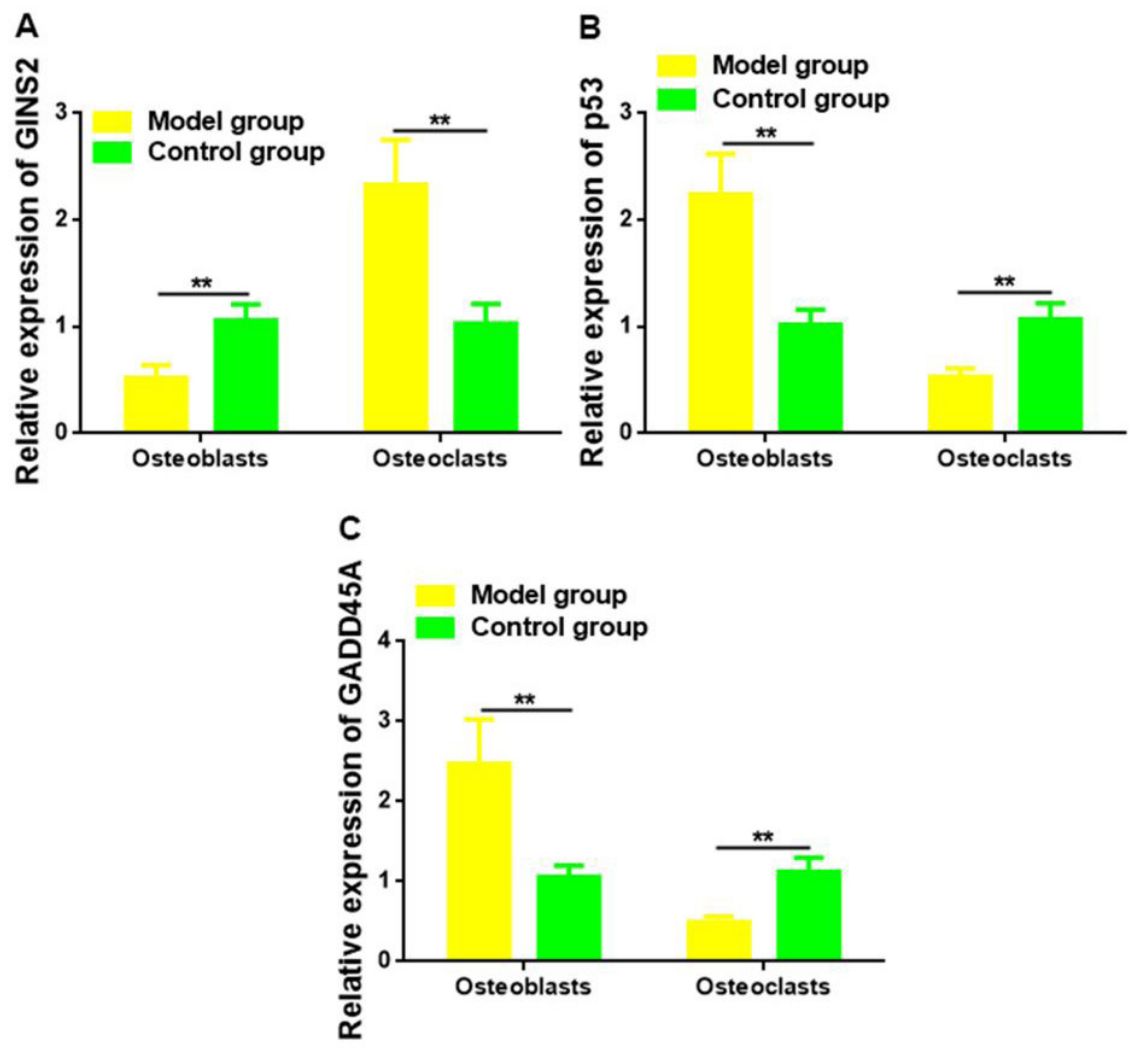

Figure 3. The relative mRNA expressions of GINS2, p53/GADD45A in osteoblasts and osteoclasts of mice. GINS2, GINS complex subunit 2; GADD45A, growth arrest and DNA damage $45 \mathrm{~A} ;{ }^{* *}$, vs Controlgroup, $\mathrm{P}<0.01$.
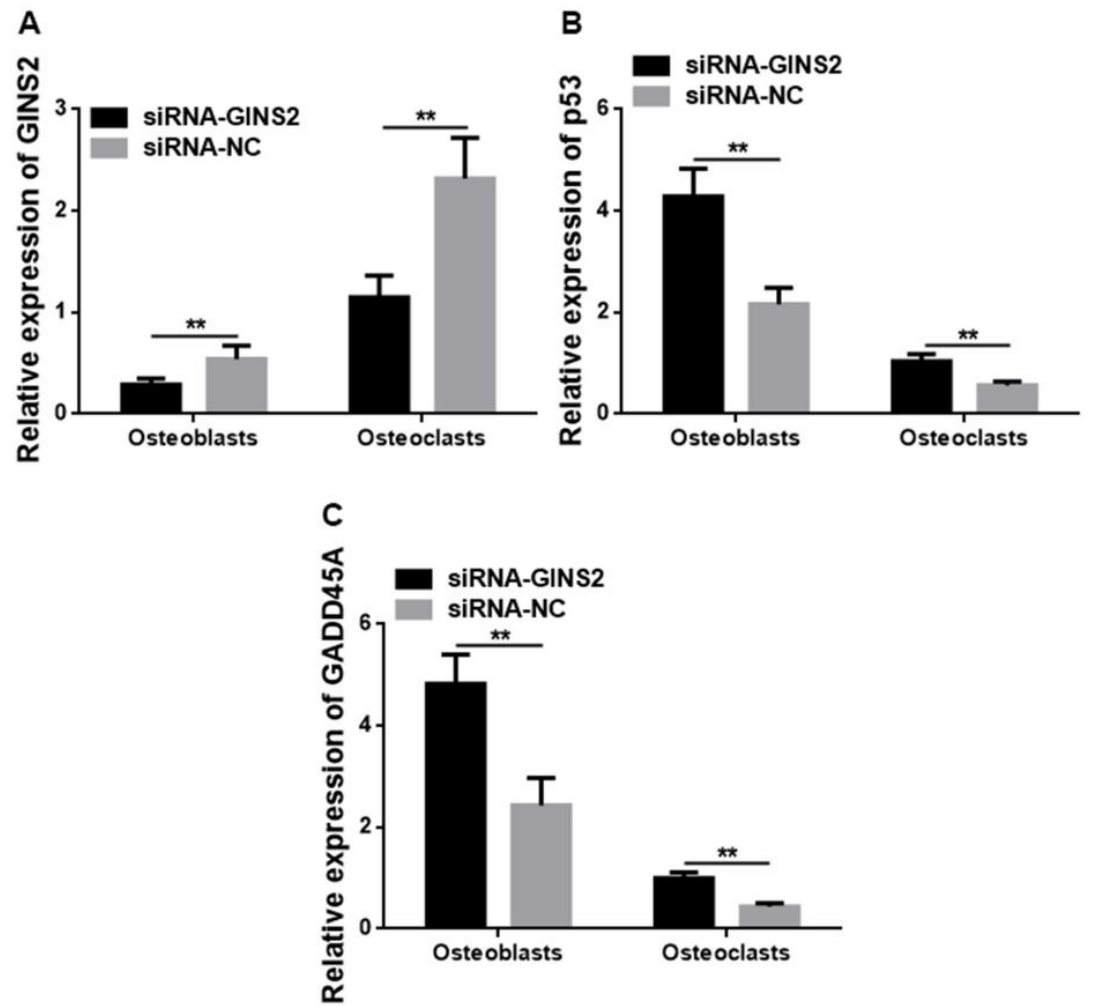

Figure 4. Down-regulated GINS2 led to up-regulations of p53 and GADD45A expressions in osteoblasts and osteoclasts of SINFH model mice. GINS2, GINS complex subunit 2; GADD45A, growth arrest and DNA damage 45A; siRNA, short interfering RNA; NC, normal control; ${ }^{* *}$, vs siRNA-NC, $\mathrm{P}<0.01$. 


\section{Discussion}

ONFH, also known as aseptic necrosis of the femoral head or ischemic necrosis of the femoral head. ONFH is a kind of pathological process, which often arises topical poor blood flow in the femoral head resulted from multiple complex causes, following with abnormal differentiation, imbalance of proportion and even death of active components in bone tissues such as osteoblasts, osteoclasts, bone marrow hematopoietic cells and subsequent repair abnormalities, resulting in further ischemia, necrosis, bone trabecular fracture and collapse of the femoral head (Mont et al., 2006; Zuo et al., 2016; Glueck et al., 2005; Hirata et al., 2007; Jones et al., 2003). Among them, steroidinduced ONFH (SINFH) is increasing year by year at clinical (Zhang et al., 2013; Yu et al., 2019), because of its unclear pathogenesis and lack of effective treatment (Wu et al., 2019), SINFH has become the focus of research in recent years. Therefore, in our study, we will explore the pathogenesis of SINFH to look for novel diagnostic and therapeutic targets.

Osteoblasts are the main functional cells of bone formation and are responsible for the synthesis, secretion and mineralization of bone matrix. The bone is constantly reconstructed. The process of bone reconstruction includes osteoclasts attached to the old bone region, secretion of acidic substances to dissolve minerals, secretion of protease to digest the bone matrix, and formation of bone resorption lacunae. Subsequently, osteoblasts migrate to the absorbed site, secrete bone matrix, and mineralize bone matrix to form new bone. The balance between osteogenic and osteogenic process is the key to maintain normal bone mass. Osteoclasts are a type of osteocyte that performs the function of bone resorption. Osteoclasts confrontat functionally to osteoblasts, and they are coordinated. Osteoclasts and osteoblasts play an important role in the development and formation of bone. When the dynamic balance between osteoblast-mediated bone formation and osteoclast-mediated bone resorption is broken, bone metabolic diseases such as ONFH, osteoporosis, osteoarthritis, etc. will develop (Wang et al., 2015, 2021). So, the abnormations of osteoclasts and osteoblasts maybe possible causes of SINFH. Moreover, during bone formation, osteoblasts must go through four phases: cell proliferation and differentiation, extracellular matrix maturation, extracellular matrix mineralization and apoptosis. Recently, Tian et al. (2020) found that enhancement of osteoblast activity and depression of osteoclast activity can ameliorate $\mathrm{ONFH}$. These evidences indicate that the proliferation, differentiation/activity, apoptosis of osteoclasts and osteoblasts may affect the occurrence and development of SINFH. Furthermore, GINS2 has been reported can facilitate cell proliferation and inhibit cell apoptosis by regulating p53/GADD45A pathway (Chi et al., 2020), but, the expression and function of GINS2 and its mechanism p53/GADD45A pathway has not been studied in SINFH, Thereby, we guess that GINS2 may affect activity/differentiation, apoptosis and proliferation of osteoblast and osteoclast to influence SINFH through activation of p53/ GADD45A signaling pathway.

In our study, we found GINS2 was up-regulated with down-regulated p53 and GADD45A expressions in the tissues at fracture surface of the head of femur in SINFH patients (Figure 1) and femoral tissues of SINFH mice (Figure 2).
Moreover, GINS2 was significantly down-regulated while p53 and GADD45A were evidently up-regulated in the process of osteoblasts formations in the SINFH mice, but in the procedure of osteoclasts formations in SINFH mice, GINS2 presented a marked upward trend while p53 and GADD45A showed a visible downward tendency compared to the control mice $(\mathrm{P}<0.01)$ (Figure 3). These finds clarified that the abnormal expressions of GINS2, p53 and GADD45A in osteoblasts and osteoclasts may a cause of SINFH. As for the source of differentiated cell lines, the mouse preosteoblast cell line MC3T3-E1 was reported a source of osteoblast (20-22), and mouse mononuclear macrophage line RAW264.7 was used as model systems of osteoclastogenesis $(23,24)$. For detecting the function and mechanism of GINS2 in SINFH, we collected the osteoblasts and osteoclasts from SINFH model mice, and transfected into siRNA-NC, siRNA-GINS2, siRNA-GINS2+siRNA-p53, siRNA-GINS2+siRNA-GADD45A, respectively. The cytoactive, proliferation and apoptosis of osteoblasts and osteoclasts were detected. The results showed that the cytoactives of osteoblasts were enhanced while the osteoclasts activities were weakened in siRNA-GINS2 group compared with siRNA-NC group $(\mathrm{P}<0.01)$ (Figure 5A), and after cotransfected into siRNA-p53 or siRNA-GADD45A, the enhanced or weakened trends were overturned partially $(\mathrm{P}<0.05)$ (Figure 5A). These findings illustrated that GINS2 has the functions of blocking MC3T3-E1 cells differentiation towards osteoblasts and inducing RAW264.7 cells differentiation towards osteoclasts. BALP is generated from osteoblasts and involved in bone formation. ALP is a homodimeric glycoprotein, and osteoblastic ALP can decompose organophosphate of cells and tissues, which provides essential phosphoric acid for the formation of hydroxyapatite and promote bone tissues mineralization. It is a specific indicator of early differentiation of osteoblasts, when osteoblasts enter the late stage, ALP activity decreases (Shidara \& Inaba, 2009). TRAP is mainly released from osteoclasts, a isoenzyme consisted in osteoclasts and considered as a marker of osteoclasts. The experiments in vitro has demonstrated that osteoclasts are active during bone resorption with a large amount of secretion of TRAP, and TRAP levels can reflect the activity of osteoclasts and the state of bone resorption (Ye et al., 2019). Our outcomes indicated that up-regulated expression of GINS2 in the tissues at fracture surface of the head of femur or femoral tissues may cause a disproportionality between osteoblasts and osteoclasts, which further breaking the dynamic balance between osteoblasts-mediated bone formation and osteoclasts-mediated bone resorption to trigger SINFH. Besides, knockdown of GINS2 expression promoted apoptosis and inhibited proliferation in both osteoblasts and osteoclasts in SINFH mice via promotion of p53/GADD45A signalling (Figure 5B-D). These outcomes indicated that abnormal expressions of GINS2 in osteoblasts and osteoclasts in SINFH may affect activity/differentiation, apoptosis and proliferation of osteoblasts and osteoclasts then cause proportional imbalance of osteoblasts and osteoclasts, which result in the occurrence of SINFH.

GINS2 has been reported over-expressed in non-small-cell lung cancer, that promotes proliferation and inhibits apoptosis by regulating the p53/GADD45A pathway (Chi et al., 2020). In addition, GINS2 also has been attested upregulated in diversiform malignancies, promoted cell proliferation, migration 

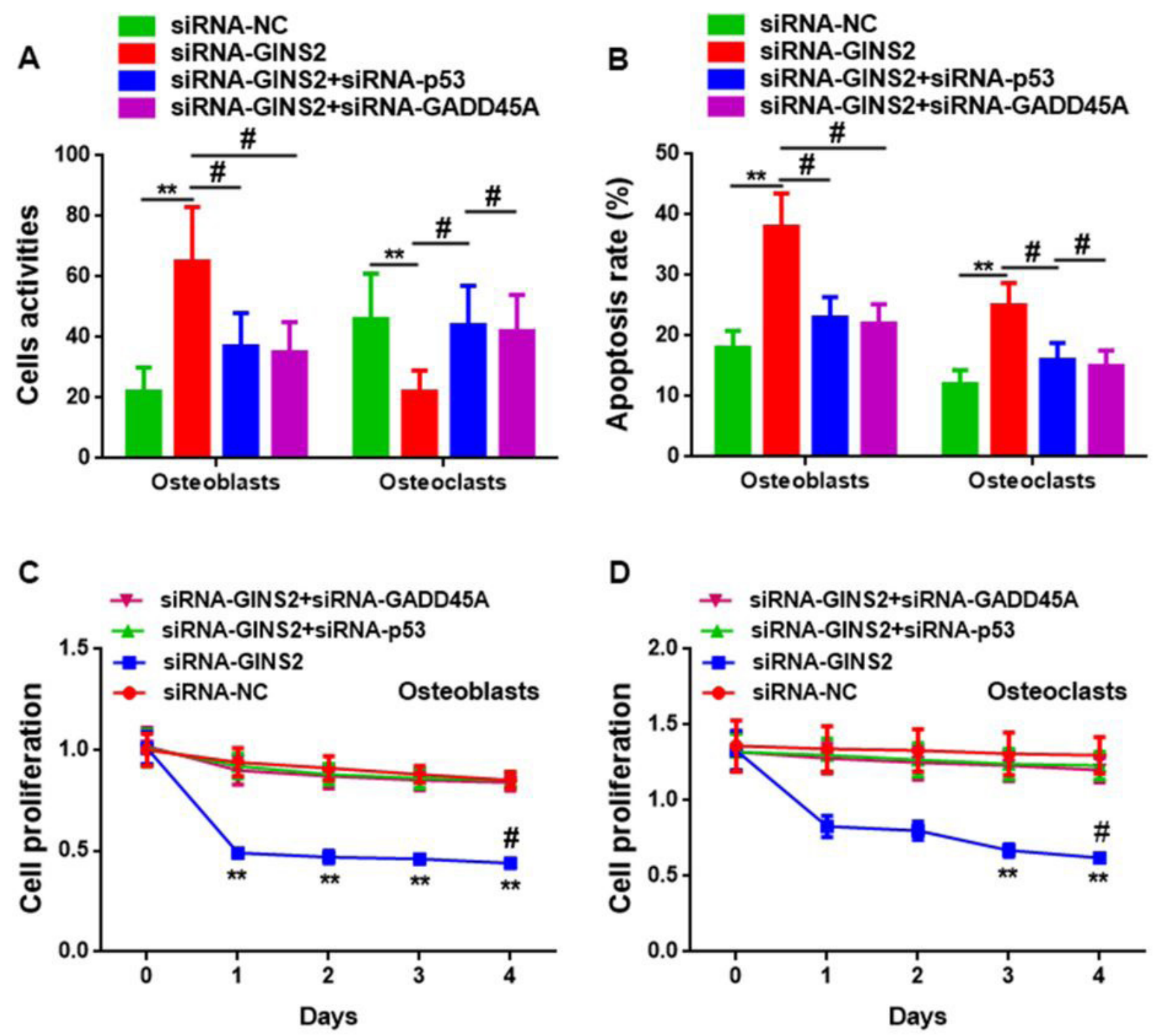

Figure 5. Knockdown of GINS2 expression increased osteoblasts activities and decreased osteoclasts activities, promoted apoptosis and inhibited proliferation in both osteoblasts and osteoclasts via promotion of p53/GADD45A signaling. (A) The activities of osteoblasts and osteoclasts in siRNA-GINS2 group, siRNA-NC group, siRNA-p53 group and siRNA-GADD45A group. (B) The apoptosis rate in osteoblasts and osteoclasts of SINFH model mice in siRNA-NC group, siRNA-GINS2 group, siRNA-p53 group and siRNA-GADD45A group. (C, D) The proliferation abilities of osteoblasts and osteoclasts in the siRNA-GINS2 group, siRNA-NC group, siRNA-GINS2+siRNA-p53 group and siRNA-GINS2+siRNA-GADD45A group. GINS2, GINS complex subunit 2; GADD45A, growth arrest and DNA damage 45A; siRNA, short interfering RNA; NC, normal control; **, vs siRNA-NC, $\mathrm{P}<0.01$; \#, vs siRNA-GINS2, $\mathrm{P}<0.05$.

and invasion, and tumor growth, inhibited cell cycle arrest and cell apoptosis, related to poor prognosis, which including thyroid cancer (Yan et al., 2018), human epithelial ovarian cancer (Ouyang et al., 2017), early-stage cervical cancer (Zheng et al., 2014), breast cancer (Grochola et al., 2010), etc. However, the expression and function of GINS2 in osteoblasts and osteoclasts in ONFH or SINFH were not been researched. The tumor inhibitor P53 has been reported suppresses cell proliferation and induces cell apoptosis, so, acts as a controlling cancer therapies (HaffnerLuntzer et al., 2018). Besides, p53 has been proved can restrain bone mass raise (Zauli et al., 2007), and inhibit proliferation and differentiation of human pre-osteoclasts (Zhang et al., 2020), restrain osteoblasts differentiation (Ohnishi et al., 2020) and advance osteoblasts apoptosis (Gu et al., 2019), and promote bone marrow mesenchymal stem cells (BMSCs) apoptosis in SINFH (Ishiguro et al., 2016). GADD45, as a downstream regulator of P53, involved in maintenance of DNA repair and cell cycle monitor of P53, which can trigger of apoptosis and inhibit proliferation (Ishiguro et al., 2016; Moriishi et al., 2014). GADD45A also has been reported can depress osteoblasts 
differentiation (Moriishi et al., 2014). These proofs strongly supportted our findings, and we are the first to report the expressions and functions of GINS2, P53 and GADD45 in osteoblasts and osteoclasts in SINFH.

\section{Conclusion}

In summary, we found that knockdown of GINS2 expression increased osteoblasts activities and decreased osteoclasts activities, promoted apoptosis and inhibited proliferation in both osteoblasts and osteoclasts via promotion of p53/GADD45A signaling pathway in SINFH. Abnormal expression of GINS2 in the osteoblasts and osteoclasts may smash the dynamic balance between osteoblasts-mediated bone formation and osteoclastsmediated bone resorption, which triggers SINFH. GINS2 and p53/GADD45A may be a potential targets for diagnosis and treatment of SINFH.

\section{References}

Amanatullah, D. F., Strauss, E. J., \& Di Cesare, P. E. (2011). Current management options for osteonecrosis of the femoral head: part 1, diagnosis and nonoperative management. American Journal of Orthopedics, 40(9), E186-E192. PMid:22022684.

Atanga, E., Dolder, S., Dauwalder, T., Wetterwald, A., \& Hofstetter, W. (2011). TNFa inhibits the development of osteoclasts through osteoblast-derived GM-CSF. Bone, 49(5), 1090-1100. http://dx.doi. org/10.1016/j.bone.2011.08.003. PMid:21884837.

Chen, J. (2018). miRNA-195 suppresses cell proliferation of ovarian cancer cell by regulating VEGFR2 and AKT signaling pathways. Molecular Medicine Reports, 18(2), 1666-1673. http://dx.doi. org/10.3892/mmr.2018.9098. PMid:29845300.

Chen, B., Du, Z., Dong, X., Li, Z., Wang, Q., Chen, G., Zhang, G., \& Song, Y. (2019). Association of variant interactions in RANK, RANKL, OPG, TRAF6, and NFATC1 genes with the development of osteonecrosis of the femoral head. DNA and Cell Biology, 38(7), 734-746. http:// dx.doi.org/10.1089/dna.2019.4710. PMid:31149839.

Chi, F., Wang, Z., Li, Y., \& Chang, N. (2020). Knockdown of GINS2 inhibits proliferation and promotes apoptosis through the p53/ GADD45A pathway in non-small-cell lung cancer. Bioscience Reports, 40(4), BSR20193949. http://dx.doi.org/10.1042/BSR20193949. PMid:32181475.

Seixas, J. T. Fo., Ide, L. K., Mello, S. C. R. P., Cafiero, J. T. G., \& Rodrigues, E. (2020). Production of flour made from bullfrog's meat and bone. Food Science and Technology, 40(Suppl. 2), 374-379. http://dx.doi. org/10.1590/fst.17419.

Glueck, C. J., Freiberg, R. A., Sieve, L., \& Wang, P. (2005). Enoxaparin prevents progression of stages I and II osteonecrosis of the hip. Clinical Orthopaedics and Related Research, \&NA;(435), 164-170. http:// dx.doi.org/10.1097/01.blo.0000157539.67567.03. PMid:15930934.

Grochola, L. F., Zeron-Medina, J., Mériaux, S., \& Bond, G. L. (2010). Single-nucleotide polymorphisms in the 53 signaling pathway. Cold Spring Harbor Perspectives in Biology, 2(5), a001032. http://dx.doi. org/10.1101/cshperspect.a001032. PMid:20452958.

Gu, X., Wang, Z., Gao, J., Han, D., Zhang, L., Chen, P., Luo, G., \& Han, B. (2019). SIRT1 suppresses p53-dependent apoptosis by modulation of p21 in osteoblast-like MC3T3-E1 cells exposed to fluoride. Toxicology in Vitro: an International Journal, 57, 28-38. http://dx.doi. org/10.1016/j.tiv.2019.02.006. PMid:30738887.
Haffner-Luntzer, M., Kovtun, A., Fischer, V., Prystaz, K., Hainzl, A., Kroeger, C. M., Krikki, I., Brinker, T. J., Ignatius, A., \& Gatzka, M. (2018). Loss of p53 compensates osteopenia in murine Mysm1 deficiency. The FASEB Journal, 32(4), 1957-1968. http://dx.doi. org/10.1096/fj.201700871R. PMid:29203593.

Hirata, T., Fujioka, M., Takahashi, K. A., Asano, T., Ishida, M., Akioka, K., Okamoto, M., Yoshimura, N., Satomi, Y., Nishino, H., Hirota, Y., Nakajima, S., Kato, S., \& Kubo, T. (2007). Low molecular weight phenotype of Apo(a) is a risk factor of corticosteroid-induced osteonecrosis of the femoral head after renal transplant. The Journal of Rheumatology, 34(3), 516-522. PMid:17143965.

Ishiguro, H., Kimura, M., Takahashi, H., Tanaka, T., Mizoguchi, K., \& Takeyama, H. (2016). GADD45A expression is correlated with patient prognosis in esophageal cancer. Oncology Letters, 11(1), 277-282. http://dx.doi.org/10.3892/ol.2015.3882. PMid:26870203.

Jones, L. C., Mont, M. A., Le, T. B., Petri, M., Hungerford, D. S., Wang, P., \& Glueck, C. J. (2003). Procoagulants and osteonecrosis. The Journal of Rheumatology, 30(4), 783-791. PMid:12672200.

Lee, S. W., Kwak, H. B., Chung, W. J., Cheong, H., Kim, H. H., \& Lee, Z. H. (2003). Participation of protein kinase C beta in osteoclast differentiation and function. Bone, 32(3), 217-227. http://dx.doi. org/10.1016/S8756-3282(02)00976-6. PMid:12667549.

Mont, M. A., Jones, L. C., \& Hungerford, D. S. (2006). Nontraumatic osteonecrosis of the femoral head: ten years later. The Journal of Bone and Joint Surgery. American volume, 88(5), 1117-1132. http:// dx.doi.org/10.2106/JBJS.E.01041. PMid:16651589.

Moriishi, T., Kawai, Y., Komori, H., Rokutanda, S., Eguchi, Y., Tsujimoto, Y., Asahina, I., \& Komori, T. (2014). Bcl2 deficiency activates FoxO through Akt inactivation and accelerates osteoblast differentiation. PLoS One, 9(1), e86629. http://dx.doi.org/10.1371/ journal.pone.0086629. PMid:24466179.

Ohnishi, T., Kusuyama, J., Bandow, K., \& Matsuguchi, T. (2020). Glut1 expression is increased by $\mathrm{p} 53$ reduction to switch metabolism to glycolysis during osteoblast differentiation. The Biochemical Journal, 477(10), 1795-1811. http://dx.doi.org/10.1042/BCJ20190888. PMid:32242617.

Ouyang, F., Liu, J., Xia, M., Lin, C., Wu, X., Ye, L., Song, L., Li, J., Wang, J., Guo, P., \& He, M. (2017). GINS2 is a novel prognostic biomarker and promotes tumor progression in early-stage cervical cancer. Oncology Reports, 37(5), 2652-2662. http://dx.doi.org/10.3892/or.2017.5573. PMid:28405687.

Park, H., No, A. L., Lee, J. M., Chen, L., Lee, S. Y., Lee, D. S., \& Yim, M. (2010). PDE4 inhibitor upregulates PTH-induced osteoclast formation via CRE-mediated COX-2 expression in osteoblasts. FEBS Letters, 584(1), 173-180. http://dx.doi.org/10.1016/j.febslet.2009.11.043. PMid:19925797.

Rajpura, A., Wright, A. C., \& Board, T. N. (2011). Medical management of osteonecrosis of the hip: a review. Hip International: the Journal of Clinical and Experimental Research on Hip Pathology And Therapy, 21(4), 385-392. http://dx.doi.org/10.5301/HIP.2011.8538.

Shidara, K., \& Inaba, M. (2009). Bone metabolic marker for osteoporosis. Japanese Journal of Clinical Medicine, 67(5), 927931. PMid:19432111.

Tian, L., Sun, S., Li, W., Yuan, L., \& Wang, X. (2020). Down-regulated microRNA-141 facilitates osteoblast activity and inhibits osteoclast activity to ameliorate osteonecrosis of the femoral head via upregulating TGF- $\beta 2$. Cell Cycle, 19(7), 772-786. http://dx.doi.org/10 .1080/15384101.2020.1731053. PMid:32089067.

Wang, J., Liu, H., \& Zhang, Q. (2019). IGF-1 polymorphisms modulate the susceptibility to osteonecrosis of the femoral head among Chinese Han population. Medicine, 98(23), e15921. http://dx.doi. org/10.1097/MD.0000000000015921. PMid:31169709. 
Wang, S., Zhou, D., \& Lin, H. (2021). Ameliorative effects of phosphorylated peptide from Antarctic krill (Euphausia superba) against $\mathrm{H}_{2} \mathrm{O}_{2}$ induced damage in MC3T3-E1 cells. Food Science and Technology. In press. http://dx.doi.org/10.1590/fst.64920.

Wang, Y., Cao, Y., Li, Y., Guo, Y., Wang, Q., Yang, M., Zhang, N., Jin, T., \& Wang, J. (2015). Genetic association of the ApoB and ApoA1 gene polymorphisms with the risk for alcohol-induced osteonecrosis of femoral head. International Journal of Clinical and Experimental Pathology, 8(9), 11332-11339. PMid:26617857.

Wu, J., Du, Y., Song, J., Dang, X., Wang, K., Wen, Y., Zhang, F., \& Liu, R. (2019). Genome-wide DNA methylation profiling of hip articular cartilage identifies differentially methylated loci associated with osteonecrosis of the femoral head. Bone, 127, 296-304. http://dx.doi. org/10.1016/j.bone.2019.06.021. PMid:31233934.

Yan, T., Liang, W., Jiang, E., Ye, A., Wu, Q., \& Xi, M. (2018). GINS2 regulates cell proliferation and apoptosis in human epithelial ovarian cancer. Oncology Letters, 16(2), 2591-2598. http://dx.doi.org/10.3892/ ol.2018.8944. PMid:30013653.

Ye, Y., Song, Y. N., He, S. F., Zhuang, J. H., Wang, G. Y., \& Xia, W. (2019). GINS2 promotes cell proliferation and inhibits cell apoptosis in thyroid cancer by regulating CITED2 and LOXL2. Cancer Gene Therapy, 26(3-4), 103-113. http://dx.doi.org/10.1038/s41417-018-0045-y. PMid:30177819.

Yu, Y., Zhang, Y., Wu, J., Sun, Y., Xiong, Z., Niu, F., Lei, L., Du, S., Chen, P., \& Yang, Z. (2019). Genetic polymorphisms in IL1B predict susceptibility to steroid-induced osteonecrosis of the femoral head in Chinese Han population. Osteoporosis International, 30(4), 871-877. http://dx.doi.org/10.1007/s00198-019-04835-9. PMid:30852631.
Zauli, G., Rimondi, E., Corallini, F., Fadda, R., Capitani, S., \& Secchiero, P. (2007). MDM2 antagonist Nutlin-3 suppresses the proliferation and differentiation of human pre-osteoclasts through a p53dependent pathway. Journal of Bone and Mineral Research, 22(10), 1621-1630. http://dx.doi.org/10.1359/jbmr.070618. PMid:17592964.

Zhang, F., Peng, W., Zhang, J., Dong, W., Wu, J., Wang, T., \& Xie, Z. (2020). P53 and Parkin co-regulate mitophagy in bone marrow mesenchymal stem cells to promote the repair of early steroid-induced osteonecrosis of the femoral head. Cell Death \& Disease, 11(1), 42. http://dx.doi.org/10.1038/s41419-020-2238-1. PMid:31959744.

Zhang, Y., Wang, R., Li, S., Kong, X., Wang, Z., Chen, W., \& Lin, N. (2013). Genetic polymorphisms in plasminogen activator inhibitor-1 predict susceptibility to steroid-induced osteonecrosis of the femoral head in Chinese population. Diagnostic Pathology, 8(1), 169. http:// dx.doi.org/10.1186/1746-1596-8-169. PMid:24135164.

Zheng, M., Zhou, Y., Yang, X., Tang, J., Wei, D., Zhang, Y., Jiang, J. L., Chen, Z. N., \& Zhu, P. (2014). High GINS2 transcript level predicts poor prognosis and correlates with high histological grade and endocrine therapy resistance through mammary cancer stem cells in breast cancer patients. Breast Cancer Research and Treatment, 148(2), 423436. http://dx.doi.org/10.1007/s10549-014-3172-7. PMid:25348432.

Zuo, W., Sun, W., Zhao, D., Gao, F., Su, Y., \& Li, Z. (2016). Investigating clinical failure of bone grafting through a window at the femoral head neck junction surgery for the treatment of osteonecrosis of the femoral head. PLoS One, 11(6), e0156903. http://dx.doi.org/10.1371/ journal.pone.0156903. PMid:27285821. 